\title{
Les siphons de décharge dans les chambres d'eau
}

\section{Wasteway regulating siphons in forebays}

\author{
PAR M. BLANCHET \\ ixgrentever aUx Etaidisssements Neyrpic
}

English synopsis, p. 579.

Les canaux découverts amenant l'eau aux usines hydro-électriques sont, en ce qui concerne l'arasement des berges, de deux types :

- Les canaux à berges horizontales qui permettent l'arrêt de l'usine sans provoquer de débordement.

- Les canaux à berges inclinces vers l'aval suivant une pente sensiblement égale à la pente de la ligne d'eau pour le débit maximum.

Dans ce dernier cas, il est indispensable, pour éviter les débordements lors des arrêts de l'usine, de disposer dans la chambre d'eau d'un ouvrage de décharge permettant de maintenir lécoulement. Ces ouvrages de décharge sont :

soit le plus souvent des déversoirs,

soit quelquefois des vannes,

soit plus rarement des siphons.

Le choix du type de l'ouvrage de décharge est fortement influencé par la sûreté du fonctionnement, car le débordement des canaux peut ètre catastrophique, le déver'soir est done le plus souvent adopté, bien qu'il soit très désavantagé du point de vue économique.

En effet, il exige pour fonctionner une surélévation importante $h$ du niveau au-dessus de la cote $\mathrm{N}$ du plan d'eau pour le fonctionnement normal des turbines (voir fig. 1). Ceci conduit d'une part à creuser lo canal pour l'écoulement du débit maximum avec le niveau $\mathrm{N}$ à la chambre d'eau et d'autre part à élever les berges pour contenir l'écoulement de ce mème débit avec le niveau $\mathrm{N}+h$ à la chambre. Les canaux étant en général à faible pente, le remous $(\mathrm{N}+h)$ se répercute très loin à l'amont.

Si, à la place du déversoir, on installe une vanne capable de maintenir le niveau à la cote $\mathrm{N}+h$, celte cote sera également celle de fonctionnement normal des turbines. Le fond et les berges du canal seront établis d'après une seule ligne d'eau, ce qui conduit approximativement à élever le fond du canal de la valeur du remous dont il a été question précédemment. On réduit ainsi la section du canal et par suite les déblais.

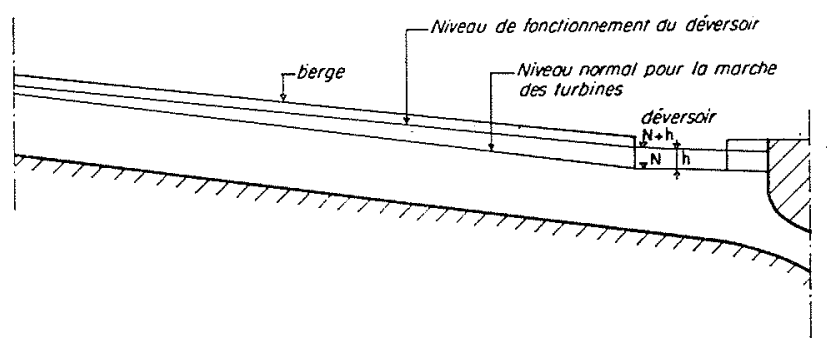

FIG. 1 .

En réalité, le problème n'est pas aussi simple, et la modification porterait sur toutes les caractéristiques du canal, telles que la section, la pente, le tracé en plan, ete... Mais finalement, la suppression du décrément $h$ donne une plus grande latitude au choix de ces caractéristiques, d'où une économie qui sera quelquefois plus importante qu'on pourrait le penser à priori.

Dans certains cas, lorsque le décrément $h$ conduit à un volume important d'eau aceumulée 
dans le canal, l'exploitation peut ètre gênée. En effet, un déclenchement à l'usine prive l'aval de ce volume d'eau et inversement une remise en marche de l'usine envoie à l'aval un excédeni de débit correspondant à ce volume.

On cherche évidemment à allonger les dévervoirs le plus possible pour réduire $h$, mais en raison des lois de débit des déversoir's, on atteint rapidement, dans cette voie, des dimensions prohibitives. Par exemple, pour un aménagement débilant $100 \mathrm{~m} \% / \mathrm{s}$, un déversoir de $100 \mathrm{~m}$ de long donne encore une surélévation $h$ de $0,60 \mathrm{~m}$ environ, soit :

-. une perte de puissance brute de 800 ch environ;

- un prix propre de l'ouvrage qui, sauf dispositions locales particulières, sera bien plus élevé que celui d'une vanno, pour peu que l'on dispose de quelques mètres de chute; - ct enfin des majorations importantes sur les travaux de terrassement.

Tous ces désavantages font qu'en pratique, lorsque les débits sont importants, on renonce au déversoir et l'on installe des vannes automatiques ou commandées par la centrale.

Entre ces deux solutions, le déversoir très sûr, mais généralement coûteux, et la vanne plus économique mais qui laisse planer le risque d'une défection de la partie mécanique ou du personnel, se place la solution siphon.

Pour jouer convenablement son rôle de déchargeur de sécurité, le siphon doit réagir de la manière suivante:

$1^{\circ}$ Il doit s'amoreer dès que le débit turbine devient inférieur au débit du canal et que le niveau, de ce fait, commence à monter au-dessus du niveau normal de marche des turbines;

$2^{\circ}$ Cet amorçage doit ètre très rapide, car lors des déclenchements, une onde positive damnlitude importante commence à remonter le canal; tout retard important dans l'amorçage laisse passer vers l'amont une part importante de l'onde, d'où un débordement possible si les berges n'ont pas été prévues pour cette onde. On doit toutefois noter que ce débordement est limité à un certain volume;

3" Après avoir absorbé l'onde, le débit du siphon doit devenir et rester égal au débit coupé par les turbines. Cet ajustement sera d'autant plus justifié si le canal aval est commun avec les turbines et n'est pas capable de débiter un supplément de débit en cas de marche intempestive du siphon. Cet ajustement du débit s'obtient par la partialisation, e'est-à-dire par une admission d'air dans le siphon, admission qui est réglée par le niveau amont. Il faut que cet ajustement soit stable et que le décrément du niveau amont soit autant que possible faible pour conserver un avantage marqué sur le déversoir;
4" Il faut enfin que le siphon ne se laisse pas obstruer par les corps flottants susceptibles d'arriver par le canal, ou ne soit pas gêné par le gel.

Toutes ces qualités, il faut évidemment les obtenir sans aucun organe mécanique mobile.

Le schéma classique de siphon se présente sous la forme de la figure 2. L'étanchéité du si-

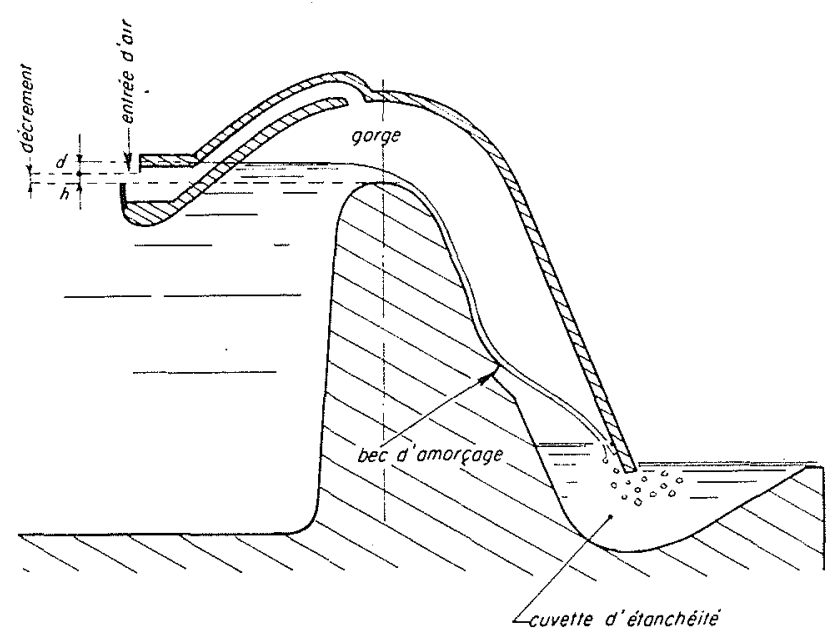

Frg. 2. - Siphon du type classique.

phon est obtenue en faisant plonger la lèvre amont dans la retenue amont, et la lèvre aval dans un bassin de restitution appelé cuvette d'étanchéité aval. L'amorçage est obtenu par le déversement de l'eau sur le seuil intérieur et par un bec d'amorcage qui dirige l'impact de la lame déversante en un point propice à l'entrainement d'air. La partialisation est obtenue par un conduit d'air dont l'orifice d'entrée est calé dans la zone du décrément choisi pour le niveau amont et dont l'extrémité aval débouche dans la gorge.

\section{Sécurité et rapidité d'amorçage}

Pour que le siphon s'amorce, il faut :

- Que le niveau amont s'élève suffisamment pour obturer l'entrée d'air de la partialisation;

- Que la cuvette aval se remplisse si elle était vide;

- Que l'air intérieur soit évacué.

Pour diminuer le retard à l'amorcage lors d'un déclenchement et éviter que l'onde positive progresse dans le canal, il faut que ces trois opérations s'effectuent très rapidement après le déclenchement.

L'onde positive de déclenchement obture très rapidement l'entrée d'air et pratiquement sans retard notable si le siphon est près du départ des conduites en charge amenant l'eau aux turbines. Pour éviter le remplissage de la cuvette aval, il 
suffit de la réaliser élanche, elle est ators praliquement toujours pleine.

Le temps total d'amoreage est done pratiquement le temps de pompage de lair interne. En réalité avec un tel dispositif on observe souvent la mise en pression, lorsque, avant déclenchement, le niveau ctait bas. En efrel, l'onde arrivant brusquement, comprime l'air par sa montée dans la branche amont du siphon, si bien que le déversement sur le seuil ne se produit pas, tant que la décompression, qui s'effectue alor's par le conduit d'air, ne sera pas finie. Pour accélérer la décompression, on doit largement dimensionner le conduit d'air. On peut également araser la branche aval juste au niveau de retenue de la cuvette, mais, dans ces conditions, on risque le désamorcage par l'aval pour certaines phases de marche du siphon, et plus particulièrement pour le début de l'amoreage pour lestruels il suffira de fables agitations du plan deau dans la cuvette pour supprimer l'étanchẻité ayat.

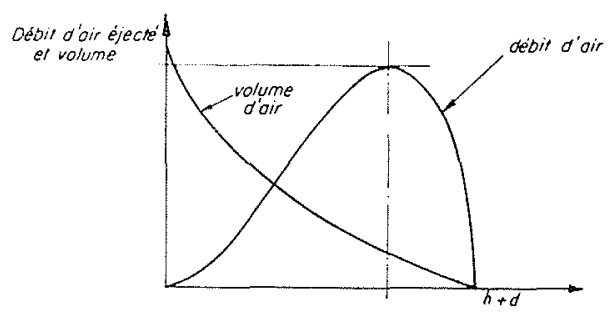

Fra. 3. - Loi de pompage d'air d'un siphon ef rolume d'air contenu dans le siphon.

Il faut, pour déterminer le temps d'amorçage du siphon, connaitre sa loi propre de pompage d'air, c'est-à-dire la variation du débit d'air éjecté a l'aval en fonction du débit d'eau du siphon ou, mieux, de la somme $h+d$, $h$ étant la hauteur du niveau dans la chambre d'eau au-dessus du seuil et $d$ la dépression dans le siphon. Cette loi se présente en général sous la forme de la figure 3. L'entraînement d'air, faible au début, croit ensuite très rapidement, passe par un maximum et redevient nul pour l'amorcage total. Dans le temps total d'amorcage, la phase la plus importante est le début car le débit d'air est tris faible et le volume à évacuer est ie plus grand. Pour réduire ce temps, on est alors conduit à placer des organes auxiliaires d'amorçage tels que des siphons auxiliaires, des éjecteurs ou tout autre dispositif hydraulique automatique. La loi idéale d'amorcage pour le problème des chambres d'eau serait celle qui donnerait un débit d'eau du siphon en fonction du temps égal à la différence entre le débit coupé aux turbines lors des déclenchements et le débit absorbé par l'onde positive dù au décrément de niveau (fig. 4). Etant donné la rapidité de fermeture des turbines, on conçoit qu il soit difficile d'obtenir une telle loi.
En réalité, les courbes de débits auront laliture sehématisée sur la figure 5 et les berges du canal devront être arasces non seulement en fonction de l'écoulement pour le niveau normal augmenté du décrément, mais encore en fonction des

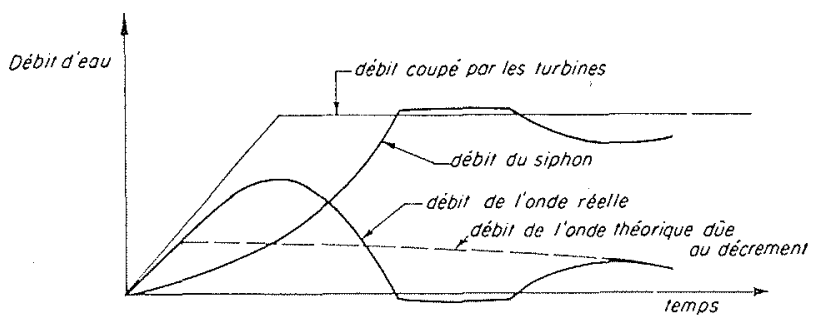

Fir. 4, - Loi idéale d'amorgage des siphons.

ondes données par la loi de débit schématisée sur la figure 5.

Il se trouve que ces ondes positives puis négatives ont un volume total relativement faible pour un amorcage rapide et qu'elles tendent à s'étaler si bien que ce n'est pratiquement que dans une zone aval limitée du canal d'amenée qu'elles interviendront dans l'arasement des berges.

Indiquons que ce problème de rapidité d'amorcage ne se pose pratiquement pas si les fermetures des turbines sont lentes, ou encore si l'usine comporte une cheminée d'équilibre qui modère suffisamment dans la chambre la diminution de débit en fonction du temps. La loi idéale d'amorçage peut alor's être aisément suivie C'est le cas par exemple du siphon du type classique, installé dans la chambre d'eau de

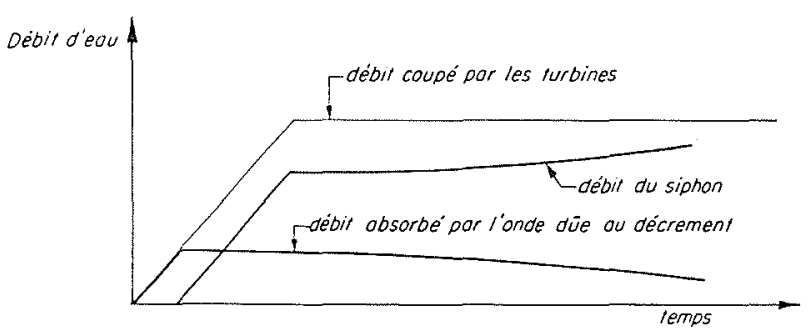

Fig. 5. - Loi réelle d'amorçage.

l'usine de Champ-sur-Drac. Le temps correspondant au pompage total de l'air contenu dans ces siphons est de l'ordre de 20 secondes.

\section{Décrément et stabilité à débit partiel}

Lorsqu'on examine la courbe de pompage d'air, on enncoit aisément qu'avec le dispositif de la figure 1 , le système puisse être stable dans la zone des débits d'air décroissants, mais qu'il n'en est pas forcément de même pour la zone des débits d'air croissants. Suivant la forme du 
siphon, l'importance de cette zone varie, mais en général la zone stable correspond au moins à un des débits d'eau compris entre les deux tiers et la totalité du débit maximum. Lorsque l'ensemble de l'ouvrage est composé d'une série de siphons indépendants, cette restriction dans la stabilité n'est pas gênante, car il suffit de décaler les niveaux d'amorcage de chaque siphon si bien que l'instabilité n'intéresse qu'une fraction du débit. Ce décalage peut porter : soit sur tous les siphons - ils sont alors tous identiques mais calés à diverses altitudes — soit seulement sur l'orifice d'entrée d'air. Ce dernier procédé nous paraît préférable car il simplifie la construction et il permet d'avoir sur tous les siphons la plus grande lame de déversante possible pour l'amorçage.

Cette manière de résoudre l'instabilité n'est toutefois possible qui si le décrément est relativement important, car il faut que les décalages soient suffisamment marqués pour éliminer des effets de vagues ou d'ondes pouvant amener brusquement le désamorçage d'un nombre important de siphons.

Aussi a-t-on cherché des dispositifs de partialisation plus stable, permettant l'emploi de décréments faibles. Les ingénieurs italiens, qui se sont toujours intéressés aux siphons et ont largement contribué à leur amélioration, ont abouti à des résultats intéressants en gardant le dispositif précédemment décrit, mais en donnant à l'entrée d'air des formes spéciales. Il semble toutefois, d'aprés notre propre expérience, que ces dispositifs ne sont pas applicables a lous les types de siphons. La Société Neyrpic a éludié et breveté un dispositif qui nous paraît résoudre d'une manière satisfaisante le probleme. Ce dispositif est rapidement décrit dans la revue ferres et Eaux, $\mathrm{N}^{\circ} 8$, à propos d'essais d'un grand siphon évacuateur de crue installé sur le barrage du Fergoug en Algérie. Il a été également appliqué aux siphons de décharge de la chambre d'eau de l'usine de la Brillanne. Ce dispositif a lavantage de servir aussi d'éjecteur auxiliaire d'amorcage.

Avec de tels dispositifs on obtient une partialisation stable à tous les débits pour de très faibles décréments, si en plus, pour les batteries de siphons, on fait un décalage des zones de réglage de chaque siphon, la partialisation et sa stabilité peuvent ètre qualifiées de parfates. Le problème est done résolu pour cette partie.

\section{Effet des corps flottants et du gel}

Les siphons doivent, pour joner leur rôle, se trouver en amont des grilles de protection des turbines; ils peuvent done recevoir tous les corps flottants amenés par le canal.

D’après les expériences que nous avons faites sur des modèles réduits de siphons et les observations dans des cas réels, nous pensons que les siphons évacuent aisément tous les corps flottants qui se présentent à leur entrée et qui ont des dimensions telles qu'il est matériellement possible de les faire passer dans les siphons, c'est-à-dire que les risques de coincement des corps sont pratiquement nuls. Le siphon ne présente en effet aucune aspérité ou rainure susceptible de favoriser des coincements et l'écoulement est tel qu'il aide constamment le corps a prendre les coudes, qui sont les passages les plus difficiles.

D’une manière générale, une protection même sommaire contre l'entrée des corps flottants à la prise d'eau suffira pour éviter tout ennui d'obturation des siphons.

Le système d'amoreage et de partialisation peut par contre être beaucoup plus gêné par l'arrivée de corps flottants. II convient d'adopter des dispositions telles que l'amorcage puisse s'effectuer totalement malgré un apport massif de corps flottants. On tolérera dans ce cas exceptionnel que la partialisation ne soit pas parfaite pourva que le siphon ne laisse pas monter le niveau au-dessus de la cote prérue, ce qui est le but principal du siphon.

Pour le problème des chambres d'eau, en effet, il n'y a pas en général d'inconvénient majeur à ce qu'exceptionnellement le siphon débite par tout ou rien. Pour cela, il suffit que l'eau puisse pénétrer dans les ouvrages d'amorcage, malgré les corps flottants, et que l'entrée d'air de la partialisation soit obturée par l'élévation normale du plan d'eau, des dispositions simples permettent d'arriver à ce résultat.

En ce qui concerne le gel, on peut craindre l'obturation aval par le gel de la cuvette aval. Nous pensons que le probleme ne peut se poser que pour des conditions climatiques locales exceptionnelles en France. En général, la couche de glace qui pourra se former sera relativement faible et très facilement disloquée par les premiers déversements qui, en remplissant la branche aval, produiront un effort pouvant etre de plusieurs tonnes par $\mathrm{m}^{2}$. Un dispositif simple pour éviter la formation de ce bouchon de glace pourra consister à établir une circulation d'eau prise dans le fond de la chambre d'eau et débitant dans la branche aval. L'apport de calories par le débit d'eau et, s'il y a lieu, l'effet de poussée par remplissage de la branche aval devrait suffire à empècher la formation d'un bouchon.

En ce qui concerne le plan d'eau dans la branche ampnt, le risque de gel ne peut exister que si le siphon est placé dans une retenue calme, qui très probablement sera déjà gelée extérieurement. On conçoit aisément que dans de telles conditions qui ne se manifestent pas brusquement, il ne peut y avoir d'eflet de surprise, d'au- 
tant plus que le personnel de la Centrale, pour d'autres raisons, devra suivre des consignes spéciales d'exploitation.

\section{Dimensionnement et forme du siphon}

Malgré certaines exigences provenant des problèmes précédemment examinés, il semble, pour le problème des chambres d'eau, que les dimensions et les formes du siphon puissent être choisies avec une très grande latitude, si bien que les problèmes d'économie, d'implantation, d'exécution auront une large place dans le choix des dimensions et formes. D'une manière générale, on aura intérèt à réduire l'encombrement en largeur de la batterie de siphons, donc à avoir un débit par mètre courant aussi élevé que possible; cela facilite l'implantation, réduit le chenal aval de restitution el diminue notablement les maconneries. A débit par mètre courant égal, il ne semble pas qu'il y ait un intérêt économique considérable à chercher un coefficient de débit particulièrement élevé exigeant des formes très continues à courbures diverses et variables. Il est souvent préférable d'avoir des formes faciles à construire, la baisse de coefficient de débit étant compenséc par une plus grande épaisseur du conduit, ce qui peut être coûteux. Cela n'est vrai que pour des siphons « peu poussés », e'est-à-dire fonctionnant assez loin des limites de cavitation, ce qui est à conseiller pour les siphons de chambre d'eau devant marcher souvent. Le probleme n'est pas le même pour les siphons évacuateurs de crues dont le fonctionnement à plein débit sera très rare et de durée relativement courte.

Compte tenu de toutes ces considérations, on obtiendra facilement des siphons pouvant débiter $10 \mathrm{~m}^{3} / \mathrm{s}$ par metre de large sous quelques mètres de chute, jusqu'à $20 \mathrm{~m}^{3} / \mathrm{s}$ pour de plus grandes installations, et cela avec une surélévation $h$ de niveau de 0,05 à $0,10 \mathrm{~m}$. Comparé à un déversoir qui donnerait seulement $1 \mathrm{~m}^{3} / \mathrm{s}$ par in pour $0,60 \mathrm{~m}$ de surélévation, ou $0,07 \mathrm{~m}^{3} / \mathrm{s}$ par $\mathrm{m}$ pour $0,10 \mathrm{~m}$ de surévélation, on voit l'avantage considírable du siphon sur l'économie du projet.

\section{Quelques applications}

Bien que les siphons ne jouissent pas d'une tres grande faveur en France, ces avantages ont été mis à profit récemment pour quelques réaménagements de chutes, consistant à augmenter le débit dérivé et à gagner sur la chute en diminuant la surélévation $h$ nécessaire au déversoir. Les craintes que l'on pouvait avoir sur ic retard de l'amorcage ou sur l'instabilité se trouvaient dans ce cas diminuées du fait de la présence du déversoir de l'installation existante,

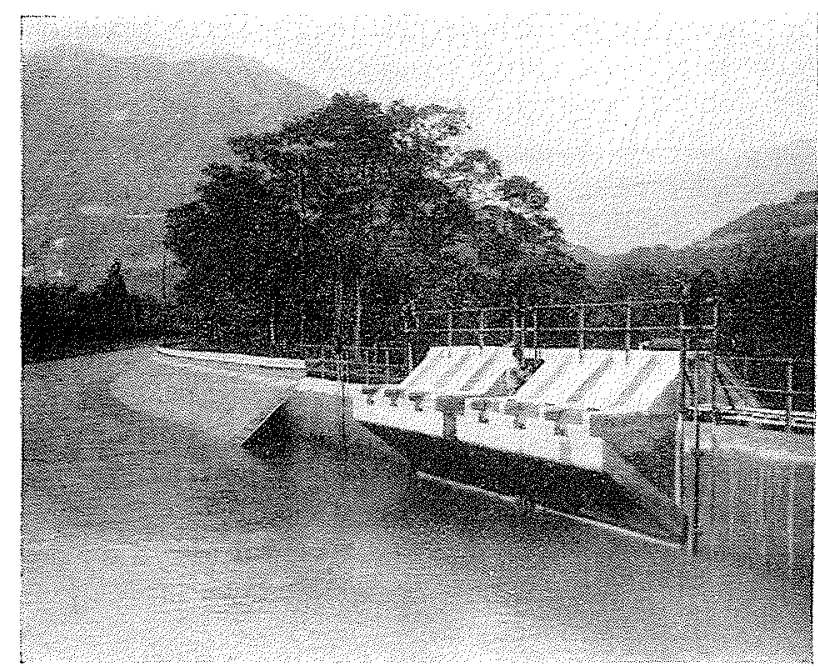

Photo $\mathrm{N}^{\circ}$ 1. - Smpoxs DE CHam-sun-Dnac. vue de la branche amont, retenue abaissede.

déversoir qui pouvait àtre maintenu en service a)rès l'avoir surélevé.

Les applications que nous connaissons plus particulièrement sont tout d'abord celles de Champ-sur-Drac où, à l'occasion de la réfection de la Centrale, le débit dérivé a été porté de 25

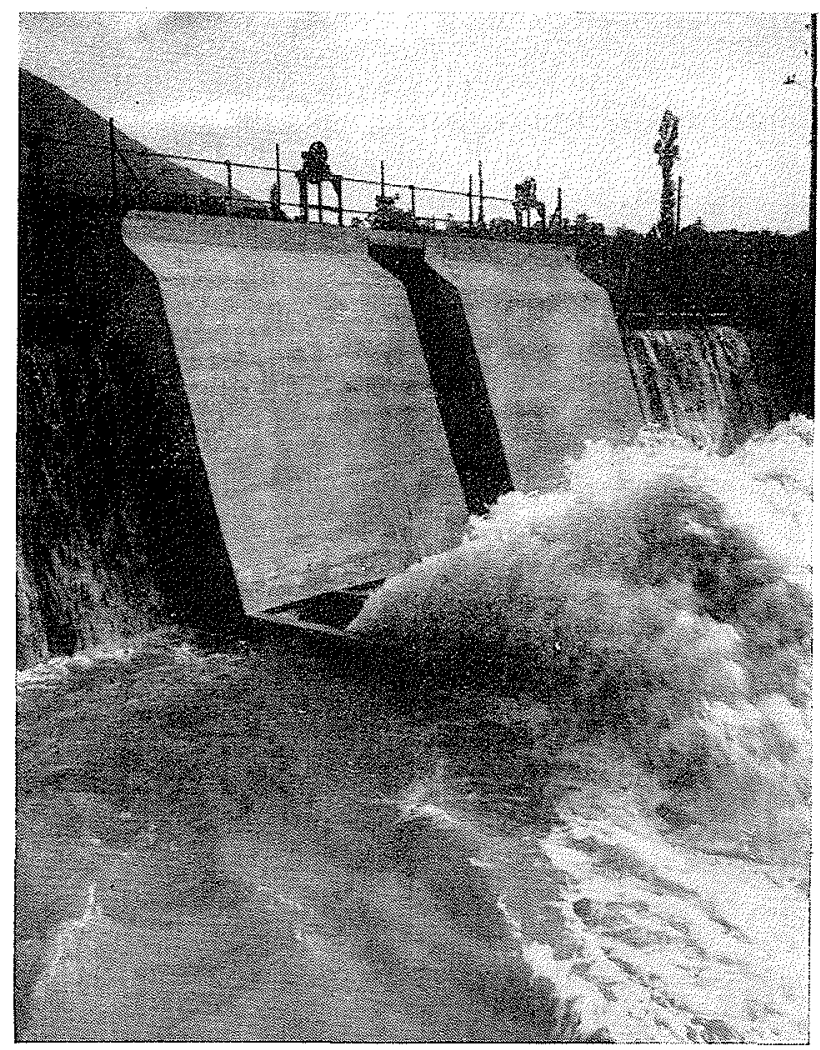

Photo $x^{\prime 2}$ 2, - Suroxs de Champ-sub-Drac. Vue d'aval pendant le fonctionnement. 
at $28 \mathrm{~m} \% \mathrm{~s}$ et la chute légèrement augmentée de $0,24 \mathrm{~m}$ par réduction du décrément.

La présence d'une cheminée d'équilibre déversante et d'un déchargeur atténuait considérablement dans le temps la montée du niveau à la chambre d'eau, si bien qu'il n'était pas néces-

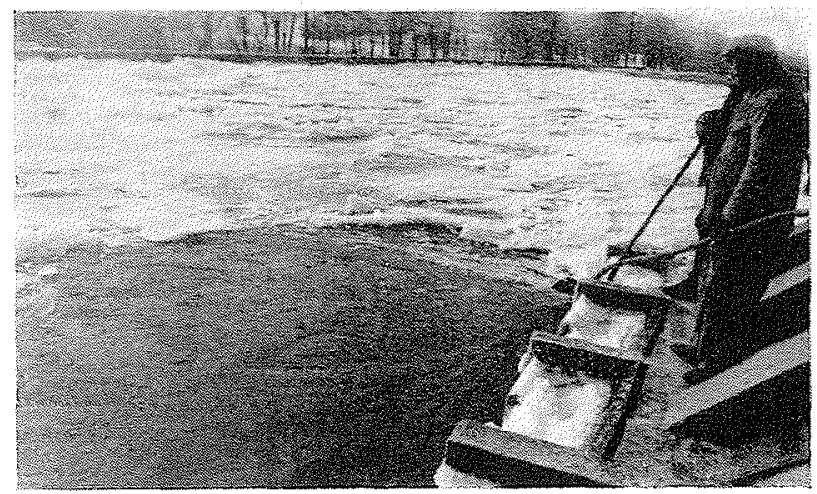

Photo $x^{\prime 3} 3$. - Siphons de Champ-sur-Drac.

Canal d'amenée encombré de glaces et sorbets. Les siphons fonctionnent à plein avec des entrées d'air bouchées par le gel, d'où un abaissement de niveau de lat retenue, ee qui est très favorable car les pertes de charge dans le canal sont augmentées par les glaces.

saire de prévoir des siphons à amorçage ultrarapide. Les siphons adoptés sont du type schématisé par les photos 1 et 2.

L'entrée du canal est protégéc par des grilles. Ces siphons sont en service depuis 1941. Les photos 3 et 4 montrent le canal et le siphon en fonctionnement un jour de gel intense et de fort charriage de glaces et de sorbets, ce qui n'est pas un cas très rare, car l'hiver la riviere est essentiellement alimentée par les lachers d'eau inter-

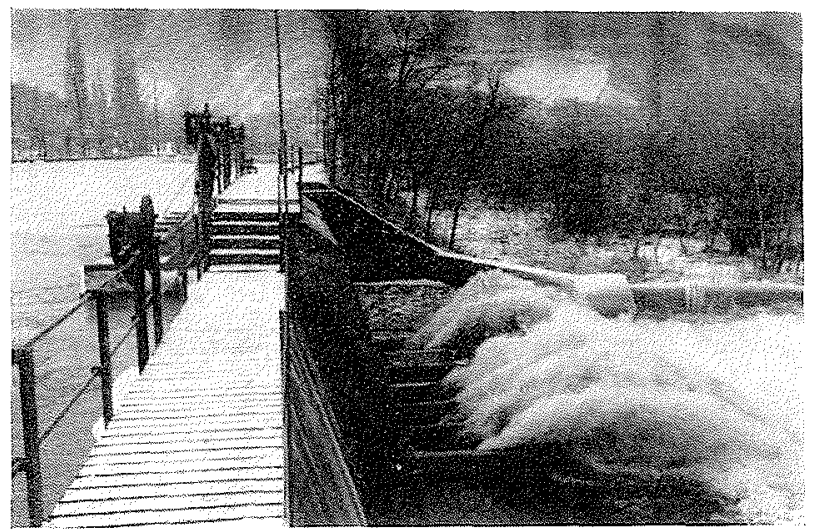

Photo ${ }^{\circ} 4$. - Smphons ine Champsth-Drac fonctionnant un jour de gel.

mittents du barrage du Sautet; ces lachers penvent se produire sur un lit à sec et enneigé. Les grilles de la prise d'eau se sont obstruées, le si- phon a joué son ròle et, de plus, contribuait à éliminer une bonne partie du sorbet et des glaces, alors que l'ancienne passe, dite à glace, était inefficace.

Les caractéristiques principales des siphons sont :

-Débit total $28 \mathrm{~m}^{3} / \mathrm{s}$ en 6 éléments hydrauliquement indépendants;

- Débit par mètre de large : $4,25 \mathrm{~m}^{3} / \mathrm{s}$;

- Chute sur la lèvre inférieure : $3,10 \mathrm{~m}$;

- Décrément total, 0,10, avec entrées d'air décalées;

- Temps d'amorçage, environ $20 \mathrm{~s}$.

Une deuxième application a été faite dans le réaménagement des chutes de la Brillanne.

Le débit dérivé était porté de 75 à $120 \mathrm{~m}^{3} / \mathrm{s}$.

Il existait un déversoir de $100 \mathrm{~m}$ de long environ et l'on a placé sur ce déversoir des siphons capables du débit total. La présence d'une vaste chambre d'eau et du déversoir autorisaient un certain retard à l'amorçage, l'onde créée par ce

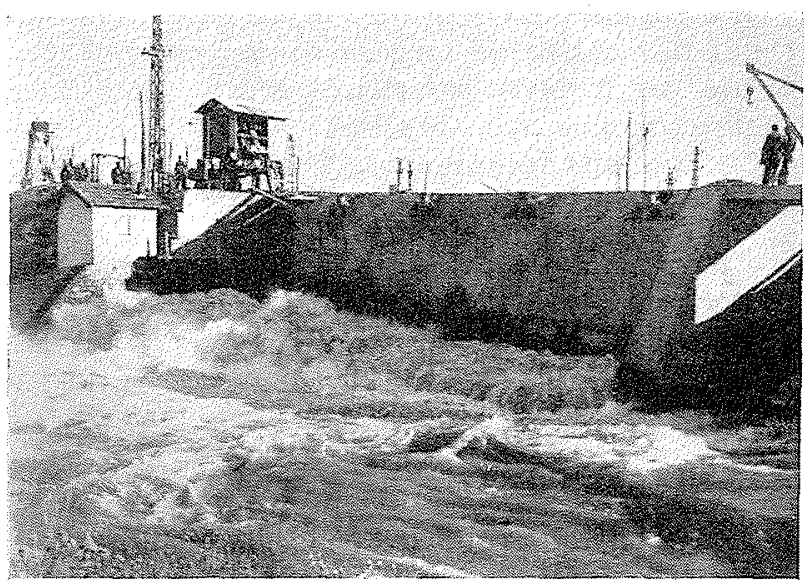

Photo $x^{\circ} 5$. - SiphoNs de la BrillanNe vus d'aval Fonctionnement en régime partiel, les siphons étant réglés pour des débits croissants de gauche à droite sur la photo.

retard pouvait ètre absorbée par la surélévation normale du niveau dù au décrément et par le léger déversement qui en résulte.

Il convenait toutefois que ce retard ne soit pas très grand. Il fallait également que la stabilité à débit particl soit très bonne, car le canal alimente trois usines en séric dont seule la plus en aval est actuellement équipéc pour le débit maximum. Les siphons doivent done marcher d'une façon presque continue en hautes eaux, pendant la phase provisoire d'équipement et pour la phase future plus fréquemment que dans les cas ordinaires, pour des raisons que l'on concoit aisément.

Ces siphons, dont le décrément total est inf'ćrieur à $0,10 \mathrm{~m}$ et dont le décrément propre à un élément est de l'ordre de 0,05 , sont écpuipés d'un 


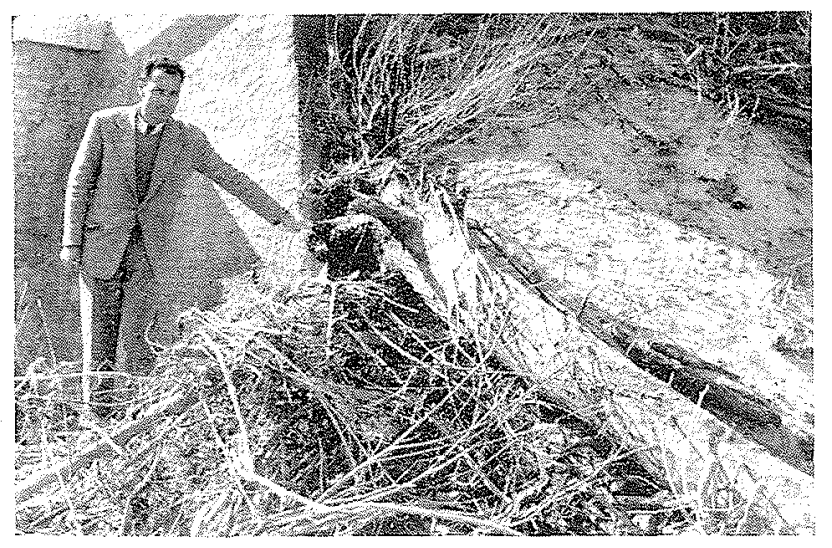

PHONO No 6. SHHONS DE LA BRHLANNE.

Exemple de corps fottant éracue par les siphons.

systeme d'amorçage et de partialisation du type Neyrpic.

Les caractéristiques principales sont :

Débit total $130 \mathrm{~m} \% / \mathrm{s}$ en 8 éléments hydrauliçuement accouplés 2 à 2 ;

Débit par mòtre de large, $8 \mathrm{~m} " / \mathrm{s}$;

Chute sur la lève inférieure, $4 \mathrm{~m}$;

Temps d'amorçage, $30 \mathrm{~s}$.

Ces siphons fonctionnent en phase provisoire, c'est-à-dire d'une manière presque continue on période de hautes eaux depuis 1948 (photo 5).

A la suite d'une très forte crue de la Durance durant une exploitation exceptionnelle de la prise d'eau, un apport considćrable de bois se produisit pendant la nuit, obstruant les grilles d'entrée des conduites forcées. Les siphons fonctionnèrent et évacuèrent une grande partie des bois (photo 6), mais une augmentation du décrément, par ailleurs sans conséquence, fut constatée. Elle était due a l'obstruction d'un écran provisoire de protection de la prise d'eau de partialisation contre les petits corps flotlants (voir photo 7), dispositif qui s'était avéré très efficace en marche continue pour les conditions normales. Sans ce dispositif, le décrément normal aurait probablement été sensiblement maintenu. Il y a licu de noter que le déversoir, qui fonetionnait sous une lame d'eau de l'ordre de $0,20 \mathrm{~m}$, était très fortement obstrué par les bois.

Les deux emplois de siphons que nous venons de signaler nous paraissent démontrer que ces appareils sont capables d'assurer la sécurité de fonctionnement de chambres d'eau alimentées par des canaux pouvant déborder par arrêt du débit à l'aval.

Dans la modernisation d'anciens aménagements ou dans les aménagements nouveaux à gros débits, nous pensons que le siphon doit prévaloir sur le déversoir très onéreux et reste avec les vannes les deux seuls dispositifs de décharge qu'il y ait lieu d'envisager.

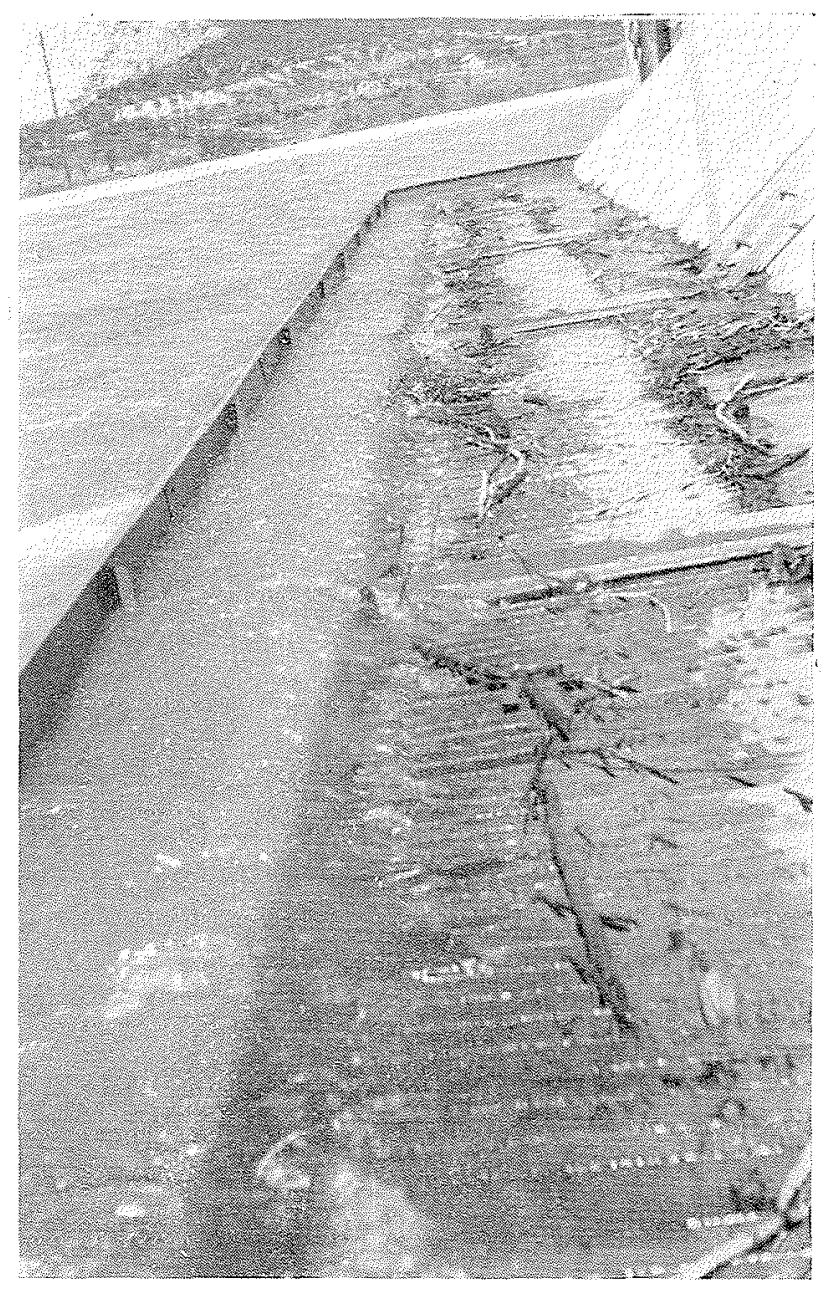

PHOTO $x^{\circ} 7$. - SipHONS DE LA BRHLANNE.

Grille d'entree du systeme d'anorçage ot de partialisation et ecran de protection des colps llotants (néfaste en cas de wes fort charriage).

\section{I S C USSION}

Sur la demande de M. le Président, M. Braxcher precise que le débit maximum a envisager pour des siphons a amorgage rapide pour chambres d'eau, est suivant la cas de 5 a $10 \mathrm{~m} 3 / \mathrm{s}$ par métre de large, ce qui équivaul a un déversoir sans siphon de plus de cinquante mètres pour le mème décrément.
Sur la demande de M Coyen, M. Brancher précise que le débil maximum des siphons évacuateurs de crue existants est par mètre de largeur de eréte de $30 \mathrm{~m}^{3} / \mathrm{s}$ pour un siphon d'essai en Algerie, et de $22 \mathrm{~m} 3 / \mathrm{s}$ pour un siphon aux Indes.

M. Corne précise que les vannes de décharge permet- 
tent actuellement des debits de $150 \mathrm{~m} \mathrm{~m}^{3 / \mathrm{s}}$ par mètre.

II. Brancher remarque que le siphon, quand il est bien construit, résoud le probleme de lautomatisme et que son prix se place entre celui des vannes qui est la solution la moins chere a installer, mais qui exige une certaine dépense de survillance et d'entretien, et celui des déversoirs trés onérenx par leur construction et la perte de chute qu'ils provoquent.

M. BLanchet montre par l'exemple d'un siphon mal construit, les défauts qu'il faut éviter pour obtenir un bon fonctionnement de ces appareils.

Sur la demande de M. Nasry, M. Buancher definit te domaine exonomique des siphons de chambre d'eau : augmentation do débit d'une chute comportant déja un déversoir, suppression des ondes par amoreage ultrilapide pour certaines usines.

Sur la demande de Mr de Sant-Vachry M. Buavenat précise qu'il est possible de régler le debit du siphon depuis le minimum jusquan maximum de facon stable. soit au moyen d'un dispositif de partialisation qui regle progressivement le debit, soit avec plusieurs siphons dont on reggle le decalage.
Sur la demande de M. Covis, M. Beancher indique que le débit du siphon par metre de larseur est pratiquement regle par la cavitation sur le seuil et en haut du conde; si l'on admet un décrement ou surcharse de montée $\gg$ de 1 à 2 mètres, il paraît possible d'ecoules $100 \mathrm{~m}^{3} / \mathrm{s}$ mais si l'on veut regler cette surcharge at $10 \mathrm{~cm}$ près, il parait difficile de depasser 50 a $60 \mathrm{~m} 3 / \mathrm{s}$; deaute part it est indispensable de connaitre les débits d'ai liecessaires et ceux-ci ne peuvent ètre déterminés qu'en construisant des siphons d'essais très importants pon les grands débits; e'est cette considération qui a ancó le Service de la Colonisation et de l'Hydraulique de l'Algeric a faire les essais du fergoug avec la collaborafion du Laboratoire Dauphinois d'Hydraulique. Le siphon du Fergoug débite $60 \mathrm{~m}^{3 /} / \mathrm{s}^{2}$ pour $2 \mathrm{~m}$ de largeur.

I. le Président ajoule que l'absorption des slaces et corps flottants, mise en relief par les pholographies projetés constitue une qualite très interessante, ef conclui que le siphon employé comme régulateur de chambre d'eau parait un précieux instrument. Il remercie vivement M. BLavemer de sal communicalion.

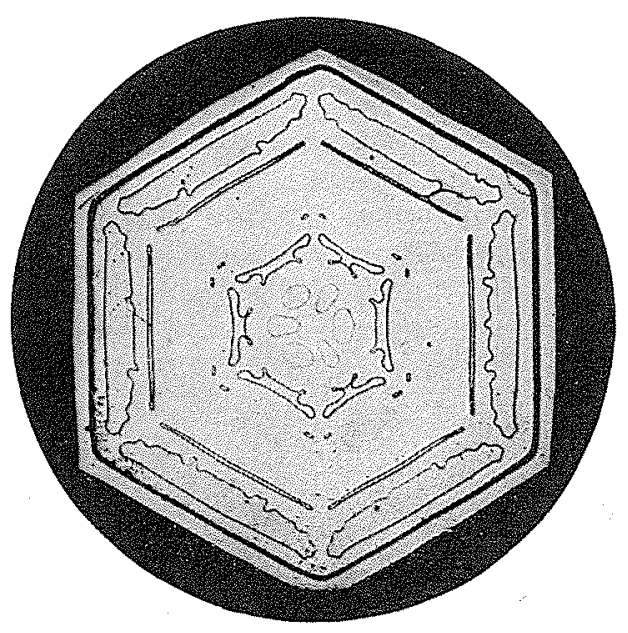

